\title{
In vitro Approaches for the Assessment of Serpin Polymerization
}

\author{
Emma L. K. Elliston, David A. Lomas and James A. Irving
}

UCL Respiratory and the Institute of Structural and Molecular Biology, University College London, WC1E 6JF

\section{Summary}

Serpin polymerization is the result of end-to-end ordered aggregation of serpin monomers into linear unbranched chains. This change in molecular state represents the basis of several conformational diseases with pathological gain-of-function and loss-of-function phenotypes, termed serpinopathies. Tools that enable quantification and characterization of polymer formation are therefore important to the study of serpin behaviour in this pathophysiological context. Such methods rely on different manifestations of molecular change: polymerization the generation of molecules with increasing molecular weight - is accompanied by concomitant structural rearrangements in the constituent subunits. Different approaches may be appropriate dependent on whether measurements are made on static samples, such as tissue or cell culture extracts, or in time-resolved experiments, often undertaken using polymers artificially induced under in vitro destabilising conditions. In the former category, we describe the application of polyacrylamide electrophoresis, western blot, ELISA and negative-stain electron microscopy; and in the latter category, Förster resonance energy transfer and fluorescence spectroscopy using environment-sensitive probes.

Key words: Polymerisation, oligomerisation, protein aggregation, monoclonal antibody, western blot, ELISA, FRET, negative-stain electron microscopy

\section{Introduction}

The ability to transition between distinct structural topologies is fundamental to the serpin mechanism, and is the product of an evolved interplay between thermodynamic instability and kinetic stability $[1,2]$. A substantial proportion of the molecule is involved in maintaining this balance [3]; consequently, there are a large number of mutations that can induce aberrant conformational behavior [4]. Such mutations are the basis of a group of conformational diseases, the serpinopathies, which result from defects in serpin secretion, turnover or from compromised functional activity. Serpinopathies can be associated with a toxic gain-of-function phenotype, from the accumulation of inactive serpin aggregates at the site of synthesis, and a loss-of-function phenotype, caused by the resultant deficiency of the active serpin [5]. These accumulating aggregates have been found to comprise linear chains of serpin molecules called polymers, which are mediated by a propagation of intermolecular interactions $[6,7]$. The ability to detect and quantify polymers is accordingly of importance in the study of the molecular and pathophysiological mechanisms that underpin several disease states.

Serpin function and malfunction are highly interrelated. Inhibitory serpins (Fig. 1A) interact with a target protease through the bait sequence in their reactive center loop. Following cleavage by the protease, the reactive center loop becomes incorporated into the 5 -stranded central $\beta$-sheet $A, a$ change 
accompanied by acquisition of thermodynamic hyper-stability. This same increase in stability is evident for polymers. Combined with the observation that peptide mimetics both stabilize the native molecule and prevent polymerization, the constituent polymer subunits have long been expected to adopt a similar 6stranded $\beta$-sheet conformation [6] (Fig. 1B). The first mechanism proposed for polymer formation directly linked the basis of this stability increase with the inter-molecular interaction: the "loop-sheet" model proposes that the reactive center loop of one molecule becomes incorporated into $\beta$-sheet $A$ of the next [6]. More recently, it has become evident that multiple polymer forms are accessible, depending on the conditions under which they form and the identity of the serpin. In crystallographic studies, different topologies have been observed for " $\beta$-hairpin" dimerized antithrombin [8] and "C-terminal" swapped trimeric antitrypsin [9]. Additionally, different destabilizing conditions have been shown to yield polymers with distinct biophysical characteristics [10-14].

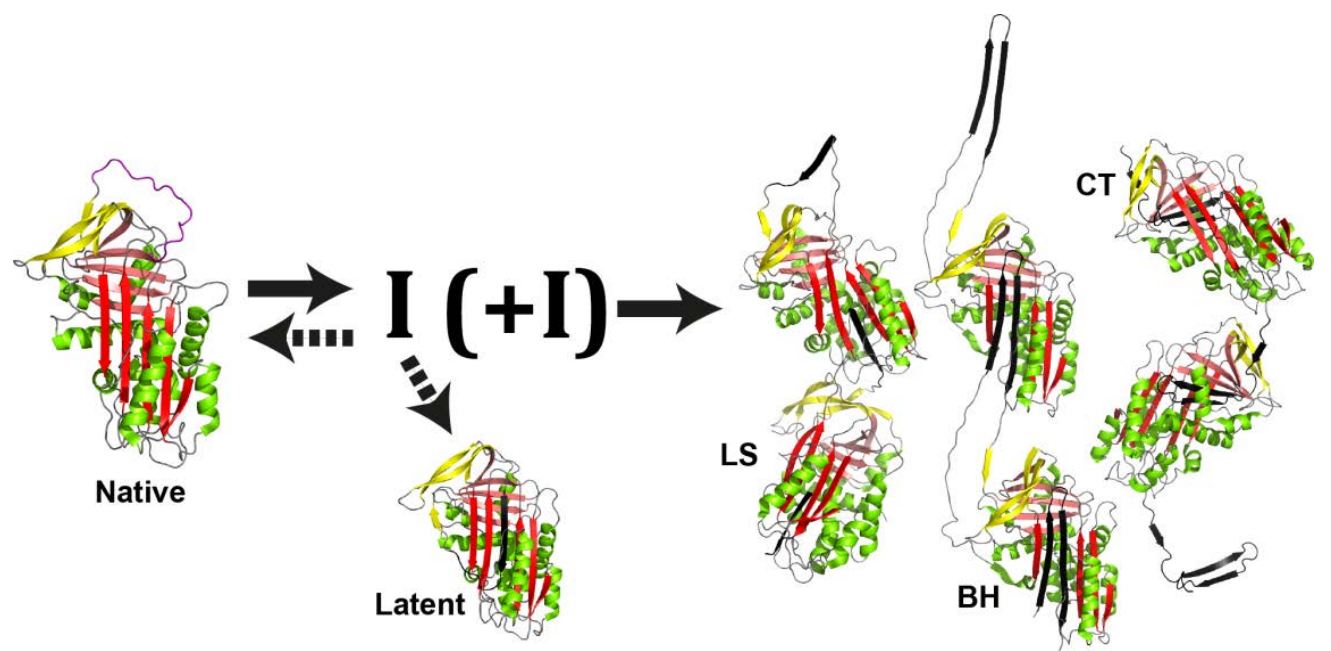

Fig. 1. A generalized reaction schematic of polymerization. Native AAT [15], representative of the core fold shared by all members of the serpin superfamily, appears at the left. Polymerization proceeds from an intermediate conformation (denoted "I"), and results initially in the formation of dimers prior to elongation into higher-order oligomers. Conformational change that occurs in the absence of oligomerization leads to the formation of a stable, misfolded latent species. There is evidence that different polymer forms are possible; models of pathological AAT polymerization have been based on biophysical (LS) and crystallographic $(\mathrm{BH}, \mathrm{CT})$ data.

The mechanism by which polymers form in general involves the adoption of a monomeric, activated intermediate state [16] which shares a cryptic epitope with the resultant polymer [17]. There is a spectrum of behavior with regards to the polymerization pathway. In neuroserpin and the $\mathrm{Z}$ mutant of alpha-1antitrypsin, this intermediate is also associated with formation of an inactive, misfolded state termed the latent conformation [18,19]. While there is no evidence of seeding with these proteins, this has been observed with alpha-1antichymotrypsin [20]. Urea-induced alpha-1-antitrypsin polymerization, which generates a markedly different polymer form to that induced by heat or found in patient tissue [10], shows some similarity to antithrombin as both polymerize predominantly through the addition of activated dimers rather than monomers [21]. 
A range of biophysical and structural techniques can be used for polymer detection and quantification, which will be outlined here with reference to the most-studied serpin in regards to polymerization, the plasma elastase inhibitor alpha-1-antitrypsin (AAT). Methods fall into two general categories: those that respond to the change in molecular size that occurs concomitantly with the formation of intermolecular interactions between serpin molecules; and those that respond to obligatory structural changes in the molecules that permit those intermolecular interactions to occur in the first place. Methods in the former category include: non-denaturing PAGE-based assays that separate polymers based on size and charge distribution, with or without detection by western blot; the use of fluorescent probes to measure inter-subunit proximity in Förster resonance energy transfer (FRET) experiments; and negative-stain EM (NSEM). The latter category includes most antibody-based assays and fluorescence spectroscopy using environment-sensitive probes.

Polymers can be isolated from tissue if this is available [6], produced in cell culture [22], or can be made artificially under thermally or chemically destabilizing conditions [20].

\section{Materials}

High purity water denotes $>10 \mathrm{M} \Omega$ resistivity, ultrapure denotes $>18 \mathrm{M} \Omega$ resistivity at $25^{\circ} \mathrm{C}$. PBS denotes phosphate buffered saline: $8 \mathrm{~g} \mathrm{NaCl}, 0.2 \mathrm{~g} \mathrm{KCl}$, $1.44 \mathrm{~g} \mathrm{Na}_{2} \mathrm{HPO}_{4}, 0.24 \mathrm{~g} \mathrm{KH}_{2} \mathrm{PO}_{4}$, made to a volume of $0.8 \mathrm{~L}$ in ultrapure water, the $\mathrm{pH}$ adjusted as necessary to 7.4 with $\mathrm{HCl}$, volume adjusted to $1 \mathrm{~L}$ with ultrapure water, and filtered through a $0.45 \mu \mathrm{m}$ filter.

\subsection{Non-denaturing polyacrylamide gel electrophoresis (PAGE)}

1. Acrylamide-based non-denaturing gel, with a Bis-Tris, Tris-Glycine or TrisTricine buffer formulation (not Tris-Borate or Tris-Acetate, however). These are readily obtainable in a pre-cast format, such as NativePAGE 3-12\% gradient gels (Life Technologies). Such systems will resolve proteins with a net negative charge at the $\mathrm{pH}$ of the electrophoresis running buffer (i.e. an isoelectric point below approximately 7). This will apply to most serpins, however those with a high isoelectric point are best resolved using an acid native PAGE system $[13,23]$, with a corresponding exchange of cathode and anode.

2. Sample buffer: $50 \% \mathrm{v} / \mathrm{v}$ glycerol, approximately $0.1 \% \mathrm{w} / \mathrm{v}$ bromophenol blue.

3. Laboratory film such as Parafilm ${ }^{\mathrm{TM}}$.

4. Sufficient running buffer to completely fill the inner (anode) chamber of the electrophoresis tank, and at minimum immerse the cathode and base of the gel in the outer (cathode) chamber.

5. Power supply capable of delivering $300 \mathrm{~V}$ or more (see Note 1)

6. Gel stain: a Coomassie blue-based stain, which can purchased commercially or be made by dissolving Coomassie Blue R-250 to a concentration of $0.1 \% \mathrm{w} / \mathrm{v}$ in a solution of $50 \% \mathrm{v} / \mathrm{v}$ methanol and $10 \% \mathrm{v} / \mathrm{v}$ acetic acid, adding the acid last.

\subsection{Western blot}


1. A non-denaturing PAGE gel that has been used to resolve sample components.

2. An electroblotting system for dry, semi-dry or wet protein transfer, such as the iBlot Dry Blotting system with associated reagents (transfer stack, cathode stack, anode stack, filter paper, disposable sponge).

3. A clean square petri dish.

4. $50 \mathrm{ml}$ polypropylene tubes.

5. Washing buffer 1: PBS pH 7.4.

6. Washing buffer 2: PBS pH 7.4, 0.1\% v/v Tween 20.

7. Blocking buffer: PBS $\mathrm{pH} 7.4,5 \% \mathrm{w} / \mathrm{v}$ non-fat dry milk powder.

8. Primary antibody buffer: washing buffer $2+5 \% \mathrm{w} / \mathrm{v}$ BSA, $0.1 \% \mathrm{w} / \mathrm{v}$ sodium azide.

9. Secondary antibody buffer: washing buffer $2+5 \% \mathrm{w} / \mathrm{v}$ nonfat dry milk.

10. Polyclonal or monoclonal primary antibody.

11. Suitable secondary antibodies, such as Odyssey donkey anti-rabbit/mouse IR secondary antibodies.

12. Detection system, such as the Odyssey IR reader.

\subsection{Sandwich ELISA}

1. 96-well EIA/RIA plates with a flat bottom and high protein-binding surface.

2. Multi-channel 20-200 $\mu$ l pipette with tips.

3. Coating buffer: PBS $\mathrm{pH}$ 7.4.

4. Washing buffer: $0.9 \% \mathrm{w} / \mathrm{v} \mathrm{NaCl}, 0.05 \% \mathrm{v} / \mathrm{v}$ Tween 20 .

5. Blocking buffer: PBS pH 7.4, 0.25\% w/v BSA, 0.05\% v/v Tween 20.

6. Blocking buffer with azide: blocking buffer $+0.1 \% \mathrm{w} / \mathrm{v}$ sodium azide.

7. Substrate solution: 3,3 ',5,5'-tetramethylbenzidine (TMB) substrate

8. Stop solution: $1 \mathrm{M}$ sulphuric acid.

9. Spectrophotometric plate reader that can read absorbance at $450 \mathrm{~nm}$.

10. Antibodies and antigens diluted in blocking buffer.

\subsection{Fluorescent labelling of serpins using amine-reactive NHS fluorophore derivatives}

1. The serpin to be labelled, purified to homogeneity.

2. A UV/visible spectrophotometer.

3. A UV transilluminator and camera, or a fluorescence gel scanner.

4. Centrifugal concentrator units with a molecular weight cut-off of $30 \mathrm{kDa}$.

5. Dialysis membrane with a cut-off between $10 \mathrm{kDa}$ and $30 \mathrm{kDa}$ (optional).

6. An amine-free buffer with a $\mathrm{pH}$ between 7.2-8.5 for conjugation such as PBS $\mathrm{pH} 7.4$ or $0.1 \mathrm{M}$ sodium bicarbonate $\mathrm{pH} 8.0$ supplemented with $0.05 \%$ sodium azide.

7. $0.1 \mathrm{M}$ hydroxylamine to quench the reaction.

8. Two NHS-ester fluorescent derivatives whose excitation and emission spectra overlap sufficiently such that the 'acceptor' fluorophore is indirectly excited upon direct excitation of the 'donor' fluorophore when they are within 50-70 $\AA$ distance (see Note 2). Combinations of fluorescein-based (such as Alexa-488 or Atto-488) and rhodamine-based (Alexa-594 or Atto594) probes work well. These are diluted to a concentration of $20-50 \mathrm{mM}$ in anhydrous DMSO and when stored in small single-use aliquots at $-80 \mathrm{C}$ are sufficiently reactive for at least 6 months. 
1. Plates and plate seal appropriate to the instrument used to monitor the fluorescence (see Note 3).

2. Two stock solutions at a concentration greater than $1 \mathrm{mg} / \mathrm{ml}$ of the serpin of interest conjugated with different fluorophores. With AAT, conjugation is often performed between a maleimidyl fluorescent derivative and the endogenous single cysteine. NHS chemistry, which primarily targets lysine residues can also be used, if optimised for a modest labelling ratio of approximately 1-2:1 fluorophore to serpin.

3. Buffer for sample dilutions such as PBS pH 7.4. For heating experiments, Tris-based buffers are best avoided due to strong effects of temperature on solution $\mathrm{pH}$.

4. For heat-induced polymerization, a real-time thermal cycler is usually required to ensure consistent and stable temperature. For chemical-induced polymerization at temperatures close to ambient, a fluorescence platereader would suffice. The instrument must be capable of specifically exciting the 'donor' fluorophore, and recording emission fluorescence of both the 'donor' and 'acceptor' fluorophores separately.

\subsection{Bis-ANS fluorescence spectroscopy}

1. Cuvette-based instrument capable of fluorescence excitation at $390 \mathrm{~nm}$ range and emission at $490 \mathrm{~nm}$ (see Note 4). For continuous monitoring of heat-induced polymerisation, this should have a Peltier temperature controller or stable heating from a circulating water bath.

2. Cuvette with excitation and emission windows, and a lid that is effective at preventing evaporation (see Note 5).

3. $20 \mathrm{mM}$ stock of 4,4'-dianilino-1,1'-binaphthyl-5,5'-disulfonic acid diluted in ethanol and stored at $-20^{\circ} \mathrm{C}$.

4. Buffer for sample dilutions such as PBS pH 7.4.

\subsection{Negative stain electron microscopy}

1. Copper electron microscopy grids with a carbon or Formvar ${ }^{\mathrm{TM}}$ support film.

2. Serpin sample diluted in a non-phosphate-based buffer (such as $50 \mathrm{mM}$ Tris $\mathrm{pH} 7.4,50 \mathrm{mM} \mathrm{NaCl}$ ) to around $0.05 \mathrm{mg} / \mathrm{ml}$.

3. Laboratory film such as Parafilm ${ }^{\mathrm{TM}}$.

4. $2 \% \mathrm{w} / \mathrm{v}$ uranyl acetate stain, diluted in high purity water and stored in the dark. Other contrast reagents can be trialled, however in our experience uranyl acetate works well.

5. Pointy-ended tweezers.

6. Grid glow-discharger.

7. Glass slide and glass petri dish.

8. Filter paper.

9. Timer.

10. Bench microfuge.

11. A low voltage (100-120kV) electron microscope and grid loader.

12. Computer with the Fiji implementation of ImageJ installed [24].

\section{Methods}

Carry out all procedures at room temperature unless otherwise specified. 


\subsection{Non-denaturing polyacrylamide gel electrophoresis (PAGE)}

Serpin polymers are typically non-covalent in nature and therefore generally not resolvable by SDS-PAGE. Non-denaturing PAGE however represents a suitable and straightforward technique for assessing the oligomeric state of a serpin sample, with a greater resolving power than analytical size exclusion chromatography (Fig. 2). In contrast to SDS-based methods, the rate of migration under an electric current is mediated by the intrinsic charge of the protein. As a result, molecular weight markers are not particularly useful, and direct comparison of the position of bands is best undertaken against control samples of known oligomeric state. This also means that comparison of absolute migration between different serpins is not possible, although comparisons between patterns of oligomer distribution should still be informative.

1. If present, remove the well comb from the top of the non-denaturing gel, while gently rinsing with water to displace residual buffer and non-reacted acrylamide. Remove any sealing tape that may be present.

2. Assemble the vertical gel electrophoresis apparatus with the gel, inner buffer chamber, buffer barrier (if required), and any other components to create a water-tight seal between anode and cathode chambers. The orientation of the gel should be as described by the apparatus manufacturer.

3. Pour the running buffer into the chambers, but not directly onto the gel to avoid distorting the wells. It is important that the chamber in contact with the top of the gel is filled such that the wells are submerged, and the buffer level in the other chamber at minimum is above the bottom of the gel. In the event that significant heating of the gel is observed during electrophoresis, filling the latter chamber to a higher level can assist with heat dissipation.

4. As non-denaturing PAGE separates both on the basis of size and intrinsic charge, like-for-like comparisons are the most meaningful and protein markers are generally not used. Consider use of controls with known or expected monomeric and oligomeric states.

5. Samples containing 2-5 $\mu$ g of protein are prepared by mixing at a 4:1 (or lower) ratio with sample buffer. This can conveniently be performed by dispensing $5 \mu \mathrm{l}$ drops of sample buffer into rows on a piece of Parafilm, and sequentially adding sample, with mixing. The maximum total volume for a typical standard midi-size gel is generally no more than $25 \mu \mathrm{l}$.

6. Carefully dispense the samples into the wells of the vertical gel. For large volumes, use long thin pipette tips and dispense close to the bottom of the well, gradually raising the tip as the well fills.

7. Connect the lid of the electrophoresis apparatus to the tank, and the leads to the power supply, paying close attention that the polarity is correct.

8. Different gel sizes, power settings and buffer systems will lead to different completion times. Therefore, close monitoring of the progress of electrophoresis is recommended initially (see Note 6). Unless otherwise recommended, power is applied at a constant 20-30 mA. Run the gel for around 40 minutes at $25 \mathrm{~mA}$ until the dye front reaches the bottom of the gel.

9. Carefully remove the gel from the casing by separating the plates with a spatula. This can be facilitated by submerging in water at the same time to reduce surface tension.

10. Transfer the gel to a dish for staining. Add sufficient (around 5-10ml) Coomassie gel stain to submerge, and incubate on a rocker. Depending on the stain used, protein bands will begin to appear after around 2-10 minutes. Generally staining is complete after 1-2 hours; following this, destain the gel 
as required, which for some commercial products can be achieved using sequential soaks in tap water (see Note 7).

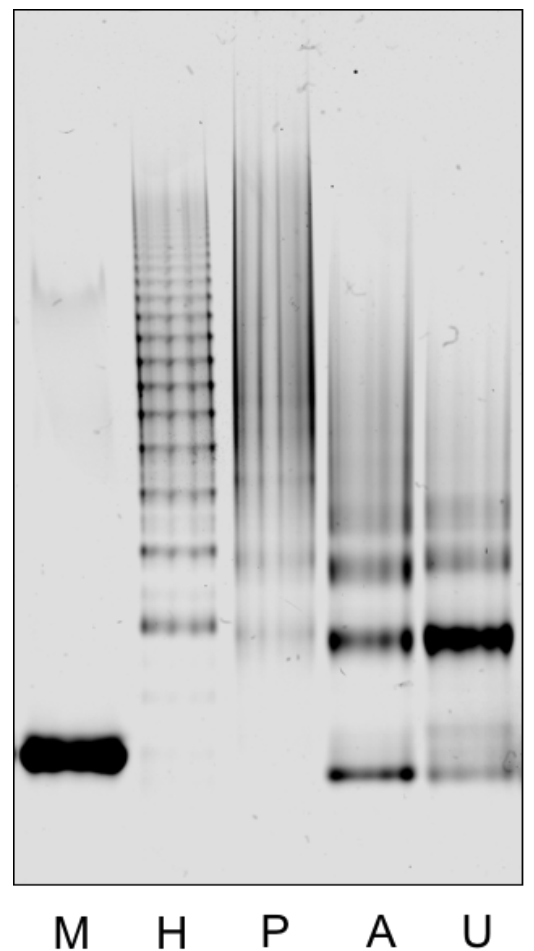

Fig. 2. A non-denaturing gel of AAT polymers formed by different in vitro methods, compared to polymers isolated from plasma. All methods produce polymers that run differently on the gel indicating that the species formed vary in size and/or charge. $\mathrm{M}$ denotes monomer; $\mathrm{H}$, heat-induced polymer; $\mathrm{P}$, plasma polymers; A, acid-induced polymer; and U indicates urea-induced polymer.

\subsection{Western Blot}

Often serpin samples are heterogenous. Coupling the resolution of nondenaturing PAGE with specific recognition by antibodies can provide a powerful means to assess oligomeric and conformational state (Fig. 3).

1. Perform non-denaturing gel electrophoresis as described in method 3.1 but load 0.2-0.4 $\mu \mathrm{g}$ of protein per well and do not stain the gel.

2. Assemble the blotting apparatus - dry, semi-dry or wet - according to manufacturers' instructions. This will involve creating a stack in which the gel is sandwiched against a PVDF membrane cut to the same size (see Note 8). These are placed between sealed buffer gel matrices in enclosed proprietary systems, or between transfer buffer-soaked filter paper and sponges for semi-dry systems and wet systems. To avoid trapping bubbles between the gel and the membrane, hold the gel (still attached to one half of the gel casing) face down and closely above the membrane. Dislodge the bottom end of the gel to touch the membrane and gently release the gel from the casing and onto the membrane little by little by lifting the casing. If necessary, gently squeeze out air bubbles by rolling a clean polypropylene tube diagonally across each layer as the 'sandwich' is built up.

3. Electroblot the gel onto the PVDF membrane as per manufacturers' instructions. 
4. Wearing new gloves, carefully transfer the membrane to a clean square petri dish and soak in washing buffer 1 for 10 minutes on a rocker before tipping away.

5. Block the membrane in $10-20 \mathrm{ml}$ blocking buffer for 1 hour on a rocker at room temperature.

6. Dilute the primary antibody to around $1 \mu \mathrm{g} / \mathrm{ml}$ in the primary antibody buffer (total volume $5-10 \mathrm{ml}$ ) and add to the membrane in a $50 \mathrm{ml}$ polypropylene tube. Incubate the membrane overnight at $4^{\circ} \mathrm{C}$ on a roller (see Note 9). Ensure the transfer side of the membrane is facing inwards, i.e. away from the sides of the tube.

7. Wash the membrane three times, each for three minutes, using washing buffer 2 .

8. Dilute the fluorescent secondary antibody at the supplier's recommended concentration in secondary antibody buffer (total volume 5-10 ml) in a new $50 \mathrm{ml}$ polypropylene tube. For Odyssey infrared antibodies, this is 1:10,000. Add the membrane, transfer-face inwards, and incubate for 1 hour on a roller at room temperature. This step (and all subsequent steps) should be carried out in the dark, to avoid photo-bleaching.

9. Wash the membrane as in step 7.

10. Wash the membrane as in step 4.

11. Image the membrane using a fluorescent scanner, such as the Odyssey IR reader.
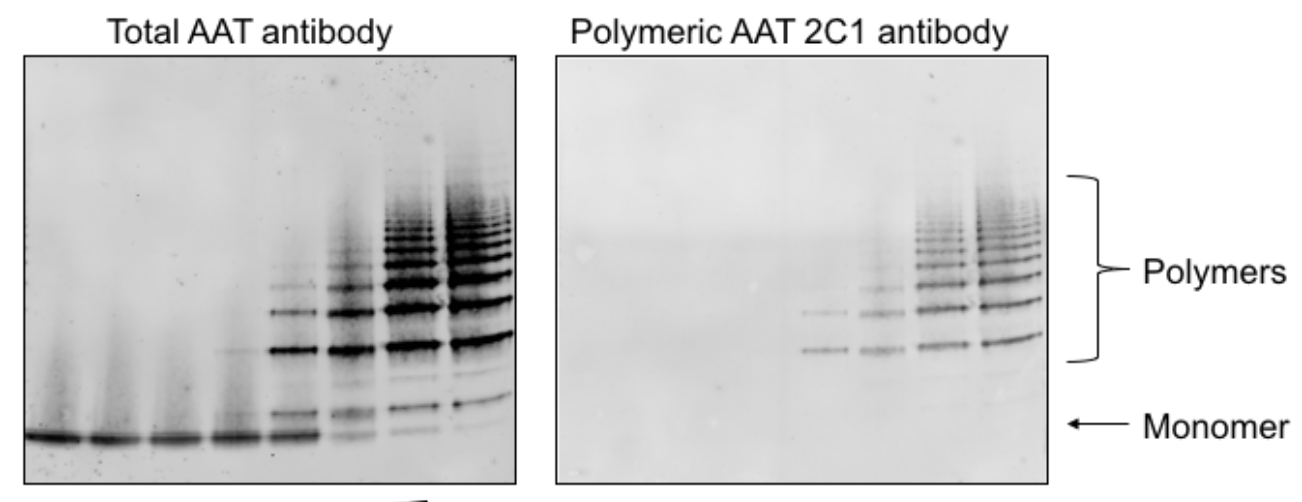

46.5

$60.5^{\circ} \mathrm{C}$

Fig. 3. Western blot of a non-denaturing gel showing the progression of AAT polymerisation at increasing temperatures. Polymeric species are only recognised by the polymer-specific antibody $2 \mathrm{C} 1$ (right panel) whereas an antiAAT polyclonal antibody recognises all conformations of AAT (left panel).

\subsection{Sandwich enzyme-linked immunosorbent assay (ELISA)}

Another antibody-based approach, the ELISA represents a complementary technique for measuring the presence of specific epitopes within a sample. In this way, the amount of serpin in a crude sample can be accurately quantified. Conformation-specific antibodies recognize cryptic epitopes; use of these reagents permit further fine-grained evaluation of conformational states within a sample (Fig. 4). Here we describe use of a sandwich ELISA technique, which uses an immobilised capture antibody to provide an in-plate enrichment of the serpin of interest. 
1. Dilute antigen-purified polyclonal anti-serpin antibody (not polyclonal sera) to $2 \mu \mathrm{g} / \mathrm{ml}$ in coating buffer.

2. Add the antibody solution to a plate at $50 \mu \mathrm{l}$ per well using a multi-channel pipette. Cover the plate with a lid to stop evaporation and allow to coat overnight on the bench.

3. Empty the plate by inverting and flicking the contents into the sink several times. Wash the plate by adding $300 \mu \mathrm{l}$ per well of washing buffer, waiting 5 minutes, then again empty the contents. Repeat the wash step twice more.

4. Add $300 \mu \mathrm{l}$ per well of blocking buffer + azide, incubate overnight at $4{ }^{\circ} \mathrm{C}$ (see Note 10).

5. Antigens (for example different polymer types) are applied with a starting concentration of $1 \mu \mathrm{g} / \mathrm{ml}$ and serially diluted 1 in 2 across the plate in blocking buffer + azide. This can be done by adding $50 \mu$ l of blocking buffer + azide to all wells except column A. In column A, add $100 \mu \mathrm{l}$ of antigen at $1 \mu \mathrm{g} / \mathrm{ml}$ in blocking buffer, aspirate $50 \mu \mathrm{l}$, mix this with the $50 \mu \mathrm{l}$ of blocking buffer in column B, aspirate $50 \mu \mathrm{l}$ and continue across the plate. Incubate the plate for 2 hours at room temperature (see Note 11).

6. Wash the plate three times as in step 3.

7. Incubate the wells with $50 \mu$ of detection (primary) antibody at $1 \mu \mathrm{g} / \mathrm{ml}$ in blocking buffer + azide for 2 hours at room temperature (see Note 12).

8. Wash the plates three times as in step 3.

9. Incubate the wells with $50 \mu \mathrm{l}$ of HRP-labelled secondary antibody diluted 1:20,000 in blocking buffer without azide. Azide inhibits the activity of HRP and so detection would not work. Incubate the HRP-labelled antibody for 1.5 hours in the dark.

10. Wash the plates three times as in step 3.

11. Develop the reaction for around 10 minutes with $50 \mu$ l of substrate solution (see Note 13).

12. Stop the reaction with $50 \mu \mathrm{l}$ of stopping solution.

13. Read the absorbance at $450 \mathrm{~nm}$ straight away in a plate reader.

14. Plot the absorbance against antigen concentration to reveal differences in affinity of the antibody for the antigens.

$2 \mathrm{C} 1$

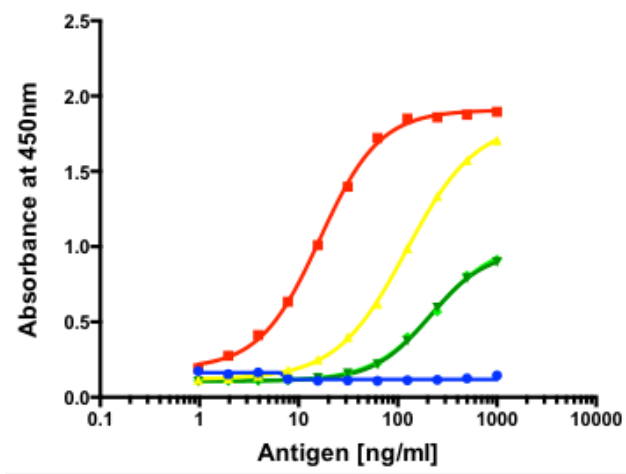

4B12

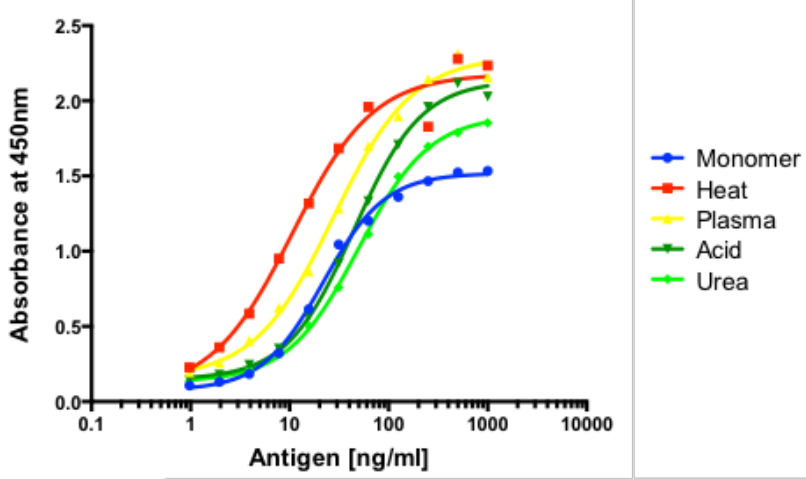

Fig. 4. Sandwich ELISA experiments using monoclonal antibodies $2 \mathrm{C} 1$ (left panel) and 4B12 (right panel) as detection antibodies against different AAT conformations. Whereas 4B12 [25] shows similar affinities for the different antigens, 2C1 [26] shows strong discrimination between different AAT polymer types.

\subsection{Fluorescent labelling of serpins using amine-reactive NHS fluorophore derivatives}


Use of FRET to monitor oligomerization requires the preparation of two serpin populations conjugated to different 'donor' and 'acceptor' fluorophores. Whilst some proteins, such as AAT, have an accessible free cysteine residue suitable for conjugation with a thiol-reactive maleimidyl group, a more widely applicable approach is the reaction between an NHS ester and surface-exposed lysines. Due to the relative abundance of this amino acid, the site of label will be distributed across the protein.

1. Buffers containing amine groups, such as Tris, are not suitable for conjugation. If required, exchange the target protein sample buffer with PBS pH 7.4 or sodium bicarbonate $\mathrm{pH} 8.0$ by dialysis, centrifugal ultrafiltration or using a desalting column. This must be thorough enough that any contaminating amine is at least below the concentration of the protein.

2. The serpin of interest should be pure and its concentration in the vicinity of $0.5-2 \mathrm{mg} / \mathrm{ml}$; adjust as appropriate using a centrifugal concentrator with a 10-30 kDa cut-off or by dilution.

3. The protein to be labelled should be divided into two tubes. Add a 5-fold molar excess of one NHS ester fluorescent probe to each. Incubate the samples at $25^{\circ} \mathrm{C}$ for 4 hours while protecting from light. These two samples should be kept separate at each step listed below.

4. Add a $1 / 10$ volume of $0.1 \mathrm{M}$ hydroxylamine to quench the labelling reaction.

5. Depending on the properties of the serpin in question, it may be possible to remove excess unconjugated fluorophore by repeating a chromatographic step used to purify it. Alternatively, sequential application to two desalting columns, or a greater than 200 -fold buffer exchange using a centrifugal concentrator unit with a $30 \mathrm{kDa}$ cut-off, should suffice.

6. The presence of unconjugated label can be evaluated by SDS-PAGE, where the free fluorophore will migrate close to the dye front. Do not stain the gel, but visualise using a UV transilluminator or fluorescence gel scanner. Using densitometry software such as ImageJ, calculate the relative intensity of the band near the dye front as a proportion of the labelled protein. Unconjugated label should be barely discernible and certainly less than $10 \%$ of the proteinbound label. If higher, perform another buffer exchange.

7. Use a spectrophotometer to determine the conjugation efficiency:

$$
\mathrm{E}=\frac{\mathrm{Abs}_{\lambda \text { max }}}{\mathrm{Ext}_{\text {label }}} \times \frac{\mathrm{Ext}_{\text {serp }}}{\mathrm{Abs}_{280 \mathrm{~nm}}-\mathrm{Abs}_{\lambda \max } * p}
$$

where $\mathrm{E}$ is the number of conjugated fluorophores per molecule of serpin, Ext $_{\text {label }}$ is the extinction coefficient of the label at $\lambda_{\max } p$ is the ratio of the absorbance of the label at $280 \mathrm{~nm}$ to its absorbance at $\lambda_{\max }$ (both parameters should be provided by the manufacturer), and Ext serp $_{\text {is }}$ the extinction coefficient of the serpin of interest. If the value of $E$ is less than 0.7 or more than 2, the labelling process should be repeated with fresh material, adjusting the excess of fluorescent conjugate accordingly.

8. Concentrate the labelled protein to at least $1 \mathrm{mg} / \mathrm{ml}$, divide into aliquots, and freeze at $-80^{\circ} \mathrm{C}$ until use.

\subsection{Förster resonance energy transfer (FRET)}

The process of polymerization brings serpin molecules into close proximity; the distances involved are on an order of magnitude that matches the size of the individual molecules (around 50-70 ) and which are amenable to use of FRET- 
based measurements. Due to the required use of specific fluorescent probes, this technique is most readily applied in vitro to purified protein, where polymerization is induced under destabilizing conditions. The progress of the reaction can be monitored on a discontinuous or continuous basis; the latter is favored for convenience and reduced intra-sample variability (Fig. 5).

1. Arrange the plate layout according to the aims of the experiment and the capabilities of the real-time thermal cycler or plate reader. For example, some thermal cyclers are able to generate a temperature gradient across the plate (see Note 14).

2. Prepare a stock mixture of the differentially-labelled serpin of interest, obtained as described in section 3.4. Based on the calculated efficiency of labelling by the two fluorophores, the relative proportions of each component should be adjusted such that the molar amount of each label is equivalent.

3. If there is no option to export raw fluorescence data from the thermal cycler or plate reader, calibrate it for the individual fluorophores according to the manufacturer's protocol. Create a non-temperature-cycling program of an appropriate length, making sure the fluorescence of both donor and acceptor fluorophores is read sufficiently frequently to capture the main features of the progress curve. On a thermal cycler, a heated lid should be used, and heating and cooling rates should be at their maximum value. Add a cooling step at the end so that the final state of the serpins can be assessed by nondenaturing PAGE.

4. For room-temperature experiments, any chilled reagents should have been brought up to ambient temperature prior to commencing the experiment.

5. If using a real-time thermal cycler, a total final concentration of the serpin (labeled and unlabeled) of $0.05-0.4 \mathrm{mg} / \mathrm{ml}$ generally works well. Dispense aliquots into wells of the PCR plate. Depending on the experimental aims, pipette additives into the relevant wells, and add the desired buffer to a final volume of $20 \mu \mathrm{l}$. A minimal volume of $40 \mu \mathrm{l}$ and $80 \mu \mathrm{l}$ is used for 384-well and 96-well plate reader microplates, respectively. Use of higher volumes may permit lower final concentrations to be used, it is important however that the non-path length-corrected absorbance of the samples at the wavelengths read is 0.1 , which may limit the upper concentration used.

6. If using a real-time thermal cycler, seal the plate with a suitable transparent film. If necessary, centrifuge the plate at $500 \mathrm{~g}$ for 1 minute.

7. Perform the polymerization experiment.

8. Export the data into a numerical package and express the 'acceptor' fluorescence as a ratio of the 'donor' fluorescence. Non-linear regression can be applied to the resulting curves; alternatively they can be normalized to values between 0 and 1.0, and the half-times at which the signals reach 0.5 can be readily determined. 

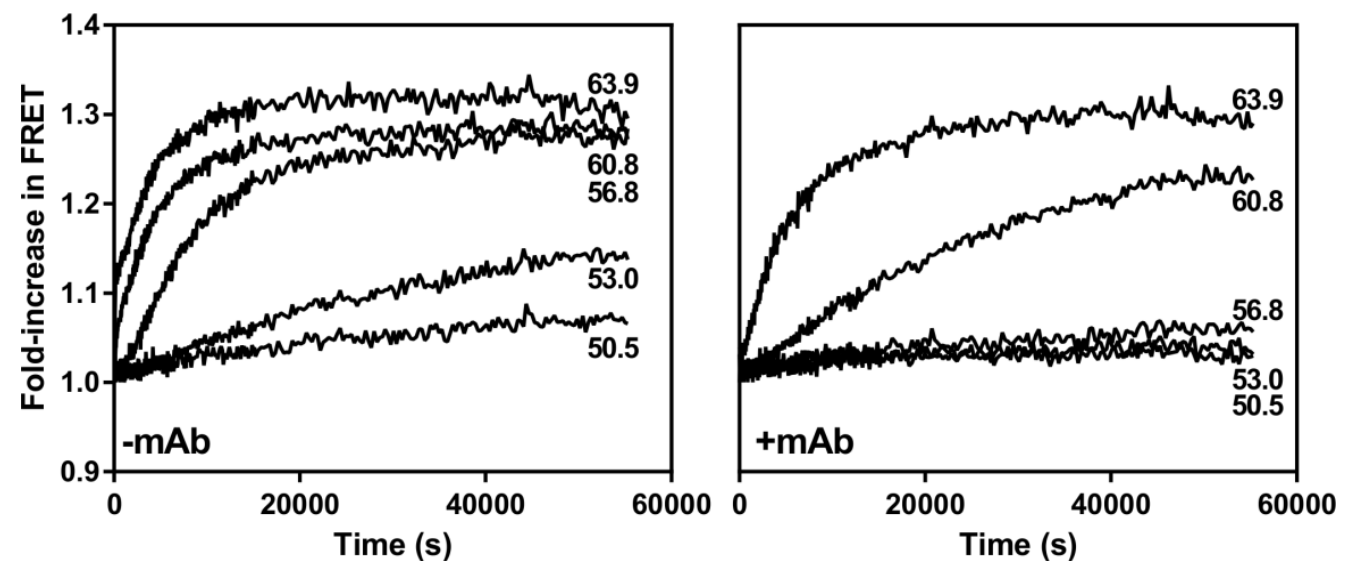

Fig. 5. FRET analysis of AAT polymerisation at the indicated temperatures using the gradient function of a real-time thermal cycler, in the absence (left panel) and presence (right panel) of a polymerisation-blocking antibody. AAT, labelled with Atto-488 and Atto-594, was incubated at a volume of $20 \mu \mathrm{l}$ and final concentration of $0.2 \mathrm{mg} / \mathrm{ml}$ in PBS. The data indicate that polymerization proceeds at a higher permissive temperature in the presence of the antibody.

\subsection{Use of bis-ANS to monitor intermediate formation}

Environment-sensitive fluorescent probes can be used to monitor changes in molecular conformation. Bis-ANS (4,4'-dianilino-1,1'-binaphthyl-5,5'-disulfonic acid) has a very low fluorescence yield in an aqueous environment, but fluoresces strongly when sequestered into a non-polar medium. In the case of AAT, bis-ANS fluorescence primarily reports the conversion from a native to polymerization-prone monomeric state, rather than the formation of polymers (Fig. 6). While in some contexts these two processes may occur almost contemporaneously, this will not be universally applicable, and therefore this distinction must be borne in mind. In addition, it has been noted that this probe has an enhanced affinity for the Z mutant of AAT [27], and can induce polymerization of plasminogen activator inhibitor-1 [28], which highlight the care that must be taken with such reagents.

1. Make the buffer that will be used for the experiment, including any additives, but do not add the serpin molecule of interest at this stage. If unsure, PBS pH 7.4 is a good general purpose buffer. Add an aliquot of the stock bis-ANS solution to give a final concentration that is at least $10 \mu \mathrm{M}$ and 5 -fold the molar concentration of the protein, whichever is higher. If intending to use a range of protein concentrations, use a consistent bis-ANS concentration across the whole experiment.

2. Prepare the spectrofluorimeter for the experiment. Add the buffer to the cuvette. If polymerization will be induced at an elevated temperature, equilibrate the heating system at the required setting, including the cuvette and as many of the components as possible (including for example buffer and oil overlay, if used). Ensure experimental parameters are set, including the length of the time-course, the frequency of data collection, and an excitation wavelength in the vicinity of $370-390 \mathrm{~nm}$ and emission wavelength of $470-500 \mathrm{~nm}$. If a magnetic flea is to be used, add this and commence stirring.

3. Add the protein of interest directly at the base of the cuvette, taking particular care if using an oil overlay. If the cuvette is not magnetically 
stirred, using a swirling motion with the tip for a few seconds to disperse the sample.

4. Conduct the polymerization experiment. The duration should be chosen to allow recording of the change in bis-ANS fluorescence to a steady-state value.

5. Repeat as required for other samples.

6. Export the data to a numerical package for analysis. With AAT, the increase in fluorescence is dominated by the first-order change from a native conformation to a polymerization-primed monomeric form, but also reports polymerization. The initial change in fluorescence corresponds with a twophase exponential equation of the form:

$$
F_{t}=F_{0}+H_{1}\left(1-\mathrm{e}^{-k_{1} \mathrm{t}}\right)+H_{2}\left(1-\mathrm{e}^{-k_{2} \mathrm{t}}\right)
$$

where $F_{t}$ is the fluorescence at time $t, F_{0}$ is the fluorescence at time $0, k_{1}$ and $k_{2}$ are rate constants of the two phases and $H_{1}$ and $H_{2}$ are the contributions of the two phases to the total signal.

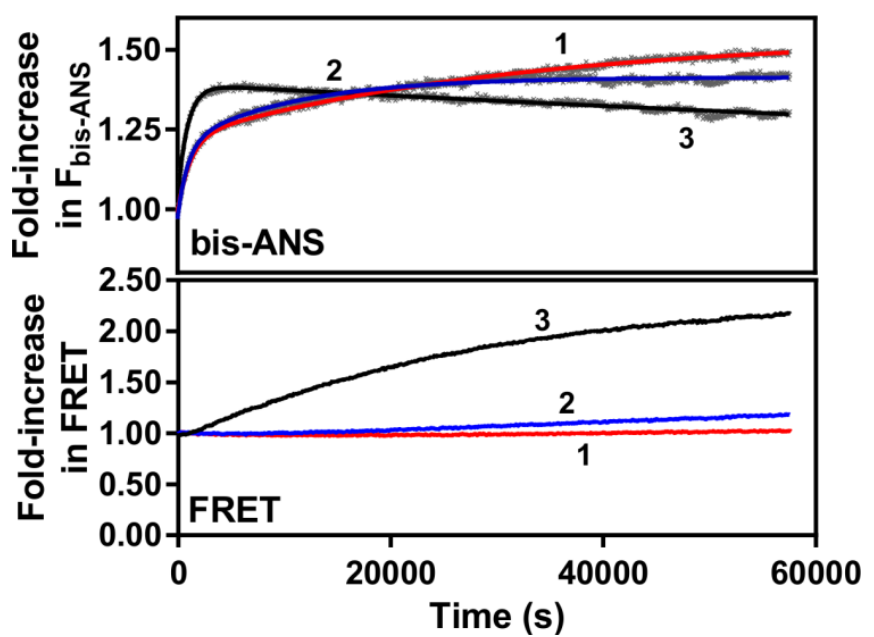

Fig. 6. Induction of AAT polymerization monitored by bis-ANS fluorescence and FRET. Curves with the same color and numbering were collected simultaneously, highlighting that the processes reported by bis-ANS (top panel) and FRET (lower panel) are different.

\subsection{Negative-stain electron microscopy}

It was this technique that first revealed polymers of AAT to be arranged as linear, unbranched chains of discrete protein subunits [6]. The use of a contrasting agent - typically, uranyl acetate - provides significantly higher contrast than achieved with cryo-electron microscopy and allows images with distinguishing features to be collected with relatively low-powered electron microscopes. Protein excludes the stain, and hence appears as light objects on a black background in the resultant images (Fig. 7). These images can be used to derive characteristics such as polymer morphology (circular/linear), chain length distribution, flexibility, inter-subunit distance and information on higherorder interactions (Fig. 8). The operation of an electron microscope is an advanced technique, and it is the preparation of the sample grids for visualization that is described here.

1. Remove copper grids from the box with the carbon side facing up using sharp tweezers and place on a glass slide. Only touch the edge of the grid with the tweezers and take care not to bend the grids. 
2. Glow-discharge the grids to make them hydrophilic by placing the glass slide in the glow discharger machine. Good parameters to start with are $30 \mathrm{~mA}$ for 1 minute and grids should be prepared shortly after glow-discharging (less than 1 hour). Transport the slide in a glass petri dish.

3. Centrifuge $2 \%$ uranyl acetate (UA) for 1 minute at $5000 \mathrm{~g}$ to pellet any precipitate.

4. Lay out Parafilm on the bench and add a $3 \mu \mathrm{l}$ drop of serpin sample diluted to around $0.05 \mathrm{mg} / \mathrm{ml}$ in a non-phosphate-based buffer such as $50 \mathrm{mM}$ Tris, $50 \mathrm{mM} \mathrm{NaCl}$. Phosphate ions can cause the precipitation of uranyl ions, which will interfere with visualisation.

5. Add two $3 \mu \mathrm{l}$ drops of the UA stain next to the sample drop from the center of the centrifuged tube.

6. Using tweezers, place the grid with the glow-discharged side face down on the sample drop and incubate for 1 minute. The sample can be incubated for longer if it is very dilute.

7. Pick up one end of the grid with the tweezers and blot off the excess sample by carefully touching the opposite edge on the filter paper.

8. Lay the grid on the first $2 \%$ UA drop and incubate for 1 minute.

9. Blot off the excess stain to leave a thin layer, and repeat the incubation and blot steps with the second $2 \%$ UA drop.

10. Leave the grids to dry face up on filter paper in a glass petri dish (see Note 15).

11. Put the grid in the grid loader of a low voltage microscope for visualization. This requires an appropriate magnification and alignments of the microscope to be carried out. Operating at relatively low magnification and higher defocus values, look for regions that show an even background stain and good dispersal of protein (light objects). Collect data from such regions at higher magnification and lower defocus values.

12. Image analysis can be performed using the Fiji implementation [24] of the ImageJ [29] software. Polymer shape and length distribution can readily be discerned. Using the fast fourier transform bandpass filter at different stringencies can help to resolve objects and allow inter-subunit distances measured (Fig. 8). 


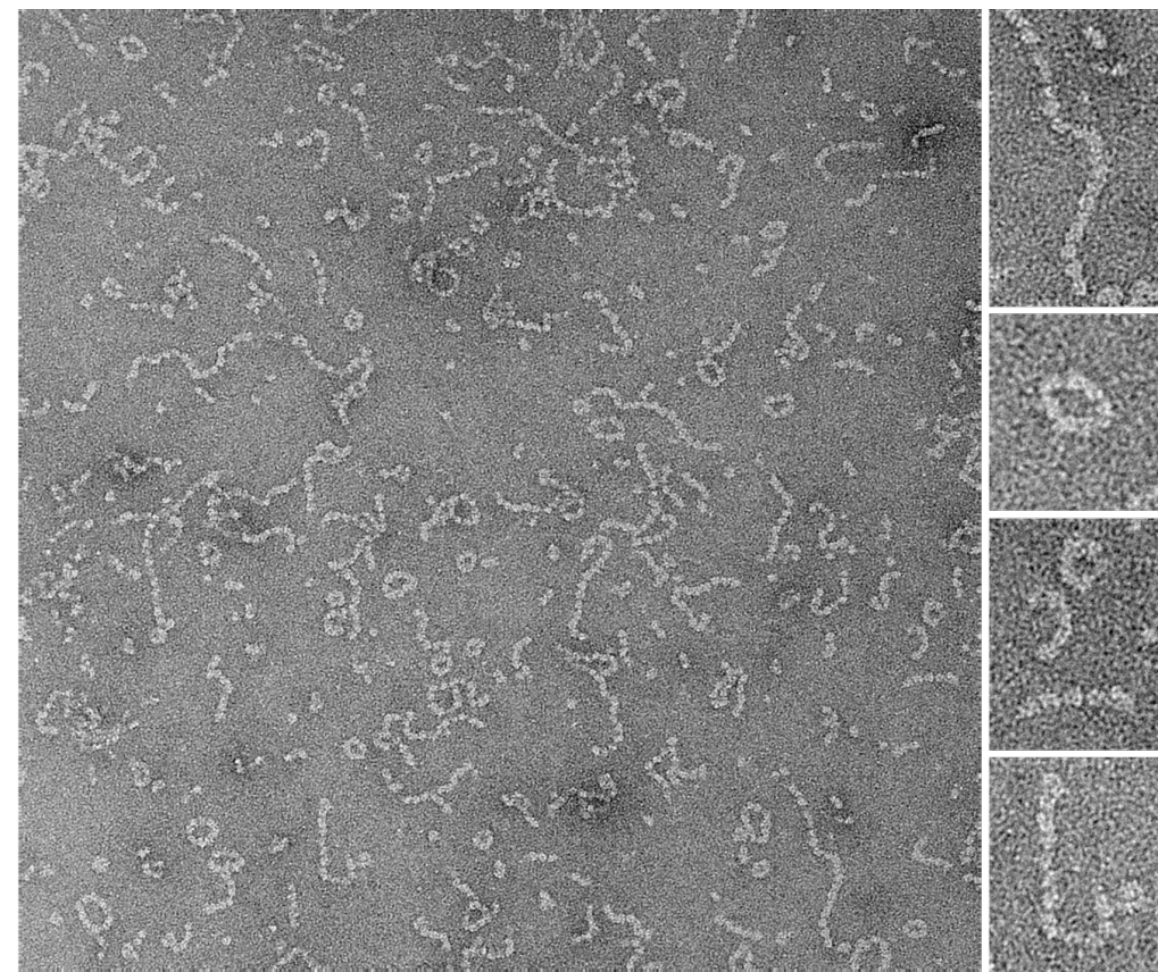

Fig. 7. A negative-stain electron micrograph of AAT polymers formed by heating monomeric AAT for 48 hours at $55^{\circ} \mathrm{C}$. Protein appears as white objects on a dark background. The grid was imaged using a Tecnai T12 equipped with a CCD camera at a nominal magnification of 67,000:1.

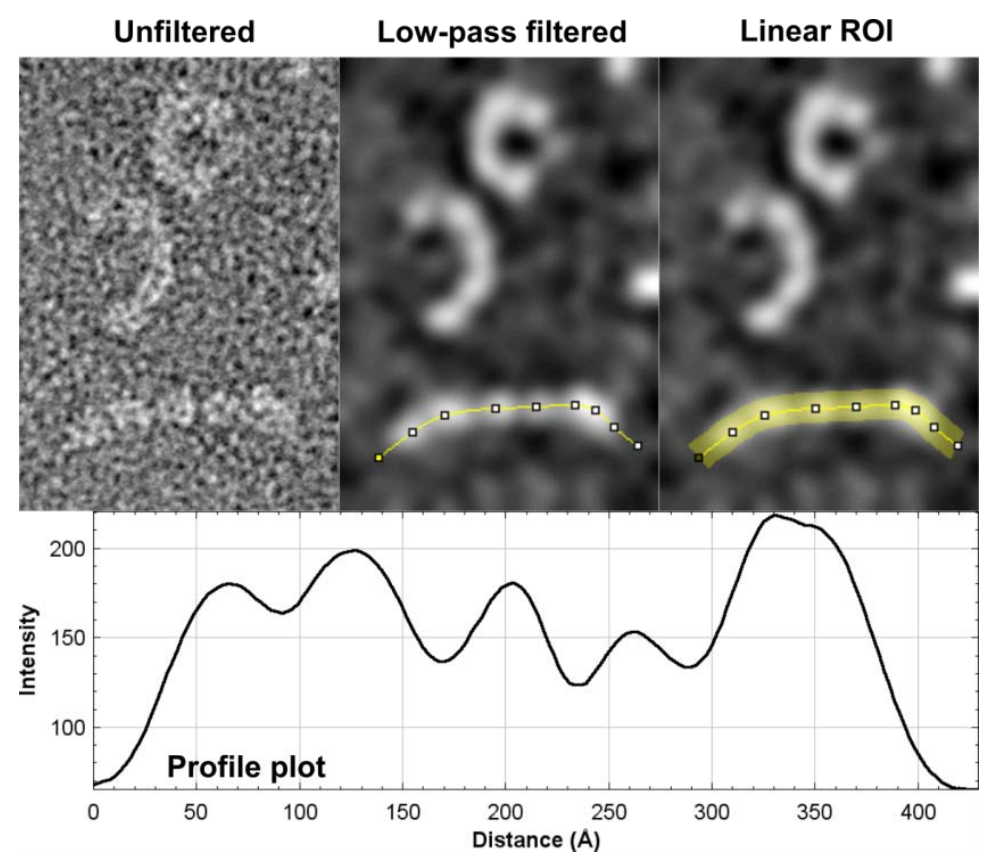

Fig. 8. Image processing of a section from a negative stain electron micrograph. A band-pass filter was applied with a stringent low-pass $35 \AA$ cutoff, a linear region of interest was defined using a smoothed spline $45 \AA$ wide, and its linearized intensity 'profile' calculated. The period of the intensity provides information on the distance between subunits in the polymer. 
1. As the resistance increases during electrophoresis, for a constant current the voltage will increase. When the voltage reaches the maximum output of the power supply, the current will drop, as will the rate of migration. Use of a power supply with a limiting voltage greater than $300 \mathrm{~V}$ will help to avoid this, however to prevent over-heating the gel it is recommended that the power output is still restricted, for Bis-Tris non-denaturing PAGE we use a limit of $15 \mathrm{~W}$ or below.

2. The Förster radius $\left(R_{0}\right)$ is the distance at which the efficiency of energy transfer is $50 \%$, and is usually reported on commercial supplier websites. In our hands, values in the vicinity of 50-70 A work well for serpin polymers.

3. White plates will tend to increase the signal output for the samples at the expense of increased background fluorescence.

4. A plate-based spectrophotometer can be used and provides the possibility of higher throughput. Differences in light path between wells due to meniscus and plate characteristics can lead to higher variability in the data for interwell comparisons. Where this option is available, excitation and emission light paths should be set to top and bottom, respectively.

5. An oil overlay can be used as a barrier to evaporation. As we have found some mineral oils can induce polymerization, we tend to use Vapor Lock (Qiagen), although other low-density fluorinated oils may also work. If using this product exercise care in cleaning the cuvette and pipetting sample; if the walls of the cuvette are wet or contaminated sample wicking may occur.

6. A NativePAGE $3-12 \%$ gradient gel (Life Technologies) run at $25 \mathrm{~mA}$ using a power supply capable of up to $3000 \mathrm{~V}$ and applying a $15 \mathrm{~W}$ limit takes about 25 minutes.

7. For home-made Coomassie stains, destaining can be undertaken by incubating with a $20 \%$ methanol, $10 \%$ acetic acid solution overnight, submerging small folds of paper towelling next to the gel to adsorb the dye.

8. Before transferring the membrane, it can be helpful to cut a little triangle off one corner so you retain your orientations of samples on the gel/membrane during washing and staining steps.

9. Two primary antibodies can be incubated with the membrane at the same time. Similarly, two Odyssey IR antibodies can then be added in the secondary antibody stage.

10. Plates can be left in blocking buffer + azide (covered with a lid) at $4{ }^{\circ} \mathrm{C}$ for several weeks.

11. Antigens can also be diluted 1 in 3 down the plate by transferring $25 \mu \mathrm{l}$ and mixing with $50 \mu \mathrm{l}$ of blocking buffer.

12. Binding antibodies need not be purified and can be added as culture medium supernatant (CMS) diluted in blocking buffer. Generally, a dilution between 1:50-1:200 is adequate depending on the secretion of the hybridoma.

13. It is best if the substrate solution is allowed to equilibrate at room temperature for 15 minutes before use.

14. The temperatures produced by the gradient function of a real-time thermal cycler do not generally increase in linear increments. To save sample while surveying the widest range of temperatures it may be appropriate to skip some columns.

15. Grids can be easily stored in a grid box for years and re-imaged. 


\section{Acknowledgement}

ELKE is the recipient of a Wellcome Trust PhD Scholarship. This work was funded by a Medical Research Council Programme Grant to DAL.

\section{References}

1. Porebski BT, Keleher S, Hollins JJ, Nickson AA, Marijanovic EM, Borg NA, Costa MG, Pearce MA, Dai W, Zhu L, Irving JA, Hoke DE, Kass I, Whisstock JC, Bottomley SP, Webb GI, McGowan S, Buckle AM (2016) Smoothing a rugged protein folding landscape by sequence-based redesign. Sci Rep 6:33958. doi:10.1038/srep33958

2. Yang L, Irving JA, Dai W, Aguilar MI, Bottomley SP (2018) Probing the folding pathway of a consensus serpin using single tryptophan mutants. Sci Rep 8 (1):2121. doi:10.1038/s41598018-19567-9

3. Seo EJ, Im H, Maeng JS, Kim KE, Yu MH (2000) Distribution of the native strain in human alpha 1-antitrypsin and its association with protease inhibitor function. J Biol Chem 275 (22):16904-16909. doi:10.1074/jbc.M001006200

4. Stein PE, Carrell RW (1995) What do dysfunctional serpins tell us about molecular mobility and disease? Nat Struct Biol 2 (2):96-113

5. Gooptu B, Lomas DA (2009) Conformational pathology of the serpins - themes, variations and therapeutic strategies. Annu Rev Biochem 78:147-176

6. Lomas DA, Evans DL, Finch JT, Carrell RW (1992) The mechanism of Z alpha 1-antitrypsin accumulation in the liver. Nature 357 (6379):605-607. doi:10.1038/357605a0

7. Davis RL, Shrimpton AE, Holohan PD, Bradshaw C, Feiglin D, Collins GH, Sonderegger P, Kinter J, Becker LM, Lacbawan F, Krasnewich D, Muenke M, Lawrence DA, Yerby MS, Shaw CM, Gooptu B, Elliott PR, Finch JT, Carrell RW, Lomas DA (1999) Familial dementia caused by polymerization of mutant neuroserpin. Nature 401 (6751):376-379

8. Yamasaki M, Li W, Johnson DJ, Huntington JA (2008) Crystal structure of a stable dimer reveals the molecular basis of serpin polymerization. Nature 455 (7217):1255-1258. doi:10.1038/nature07394

9. Yamasaki M, Sendall TJ, Pearce MC, Whisstock JC, Huntington JA (2011) Molecular basis of alpha1-antitrypsin deficiency revealed by the structure of a domain-swapped trimer. EMBO Rep 12 (10):1011-1017. doi:embor2011171 [pii]10.1038/embor.2011.171

10. Ekeowa UI, Freeke J, Miranda E, Gooptu B, Bush MF, Perez J, Teckman J, Robinson CV, Lomas DA (2010) Defining the mechanism of polymerization in the serpinopathies. Proc Natl Acad Sci U S A 107 (40):17146-17151. doi:10.1073/pnas.1004785107

11. Tsutsui Y, Kuri B, Sengupta T, Wintrode PL (2008) The structural basis of serpin polymerization studied by hydrogen/deuterium exchange and mass spectrometry. J Biol Chem 283 (45):30804-30811. doi:10.1074/jbc.M804048200

12. Devlin GL, Chow MKM, Howlett GJ, Bottomley SP (2002) Acid denaturation of a1-antitrypsin: characterization of a novel mechanism of serpin polymerization. J Mol Biol 324:859-870

13. Zhou A, Faint R, Charlton P, Dafforn TR, Carrell RW, Lomas DA (2001) Polymerization of plasminogen activator inhibitor-1. J Biol Chem 276 (12):9115-9122

14. Pedersen KE, Einholm AP, Christensen A, Schack L, Wind T, Kenney JM, Andreasen PA (2003) Plasminogen activator inhibitor-1 polymers, induced by inactivating amphipathic organochemical ligands. Biochem J 372:747-755

15. Elliott PR, Abrahams JP, Lomas DA (1998) Wild-type alpha 1-antitrypsin is in the canonical inhibitory conformation. J Mol Biol 275 (3):419-425 
16. Dafforn TR, Mahadeva R, Elliott PR, Sivasothy P, Lomas DA (1999) A kinetic mechanism for the polymerization of alpha1-antitrypsin. J Biol Chem 274 (14):9548-9555

17. Irving James A, Miranda E, Haq I, Perez J, Kotov Vadim R, Faull Sarah V, Motamedi-Shad N, Lomas David A (2015) An antibody raised against a pathogenic serpin variant induces mutant-like behaviour in the wild-type protein. Biochemical Journal 468:99-108. doi:10.1042/BJ20141569

18. Tan L, Perez J, Mela M, Miranda E, Burling KA, Rouhani FN, DeMeo DL, Haq I, Irving JA, Ordonez A, Dickens JA, Brantly M, Marciniak SJ, Alexander GJ, Gooptu B, Lomas DA (2015) Characterising the association of latency with alpha(1)-antitrypsin polymerisation using a novel monoclonal antibody. Int J Biochem Cell Biol 58:81-91. doi:10.1016/j.biocel.2014.11.005

19. Chiou A, Hagglof P, Orte A, Chen AY, Dunne PD, Belorgey D, Karlsson-Li S, Lomas DA, Klenerman D (2009) Probing neuroserpin polymerization and interaction with amyloid-beta peptides using single molecule fluorescence. Biophys J 97 (8):2306-2315. doi:10.1016/j.bpj.2009.07.057

20. Crowther DC, Serpell LC, Dafforn TR, Gooptu B, Lomas DA (2003) Nucleation of a1antichymotrypsin polymerisation. Biochemistry 42 (8):2355-2363

21. Zhou A, Carrell RW (2008) Dimers initiate and propagate serine protease inhibitor polymerisation. J Mol Biol 375 (1):36-42. doi:10.1016/j.jmb.2007.10.055

22. Miranda E, McLeod I, Davies MJ, Pérez J, Römisch K, Crowther DC, Lomas DA (2008) The intracellular accumulation of polymeric neuroserpin explains the severity of the dementia FENIB. Hum Mol Genet 17:1527-1539

23. Irving JA, Shushanov SS, Pike RN, Popova EY, Bromme D, Coetzer TH, Bottomley SP, Boulynko IA, Grigoryev SA, Whisstock JC (2002) Inhibitory activity of a heterochromatinassociated serpin (MENT) against papain-like cysteine proteinases affects chromatin structure and blocks cell proliferation. J Biol Chem 277 (15):13192-13201

24. Schindelin J, Arganda-Carreras I, Frise E, Kaynig V, Longair M, Pietzsch T, Preibisch S, Rueden C, Saalfeld S, Schmid B, Tinevez JY, White DJ, Hartenstein V, Eliceiri K, Tomancak P, Cardona A (2012) Fiji: an open-source platform for biological-image analysis. Nat Methods 9 (7):676-682. doi:10.1038/nmeth.2019

25. Motamedi-Shad N, Jagger AM, Liedtke M, Faull SV, Nanda AS, Salvadori E, Wort JL, Kay CW, Heyer-Chauhan N, Miranda E, Perez J, Ordonez A, Haq I, Irving JA, Lomas DA (2016) An antibody that prevents serpin polymerisation acts by inducing a novel allosteric behaviour. Biochem J 473 (19):3269-3290. doi:10.1042/BCJ20160159

26. Miranda E, Perez J, Ekeowa UI, Hadzic N, Kalsheker N, Gooptu B, Portmann B, Belorgey D, Hill M, Chambers S, Teckman J, Alexander GJ, Marciniak SJ, Lomas DA (2010) A novel monoclonal antibody to characterize pathogenic polymers in liver disease associated with alpha1-antitrypsin deficiency. Hepatology 52 (3):1078-1088. doi:10.1002/hep.23760

27. Knaupp AS, Levina V, Robertson AL, Pearce MC, Bottomley SP (2010) Kinetic instability of the serpin Z alpha1-antitrypsin promotes aggregation. J Mol Biol 396 (2):375-383. doi:10.1016/j.jmb.2009.11.048

28. Egelund R, Einholm AP, Pedersen KE, Nielsen RW, Christensen A, Deinum J, Andreasen PA (2001) A regulatory hydrophobic area in the flexible joint region of plasminogen activator inhibitor-1, defined with fluorescent activity-neutralizing ligands. Ligand-induced serpin polymerization. J Biol Chem 276 (16):13077-13086

29. Schneider CA, Rasband WS, Eliceiri KW (2012) NIH Image to ImageJ: 25 years of image analysis. Nat Methods 9 (7):671-675 